\title{
Structural Analysis of an Extracellular matrix protein adhesin (EmaA) of Actinobacillus actinomycetemcomitans
}

\author{
Chunxiao Yu*, Christopher Lenox*, Keith Mintz*, Teresa Ruiz** \\ * Dept. of Microbiology and Molecular Genetics, University of Vermont, Burlington, VT 05405 \\ ** Dept. of Molecular Physiology and Biophysics, University of Vermont, Burlington, VT 05405
}

The binding of bacteria to collagen is an established virulence determinant. Recently, emaA (extracellular matrix protein adhesin A) was identified as a collagen binding protein of Actinobacillus actinomycetemcomitans, a bacterium associated with localized aggressive periodontitis and other systemic human infections [1,2,3]. emaA encodes a $201 \mathrm{kDa}$ outer membrane protein and, based on primary and secondary protein predictions, belongs to a family of oligomeric coiled-coil adhesins (Oca) [4]. EmaA contains all of the conserved structural elements of the members of this adhesin family: a) an N-terminal secretion signal, b) a head domain containing the interactive site, c) a highly conserved neck region, d) a stalk domain with high coiled-coil formation probability, and e) a conserved membrane anchor domain. The N-terminal head domain of EmaA contains twelve putative collagen binding motifs, which are hypothesized to be essential for collagen binding.

Our electron microscopy studies from negatively stained preparations of whole mount bacteria have shown that EmaA forms antenna-like appendages on the surface of $A$. actinomycetemcomitans. These structures are not present on the surface of an $\mathrm{emaA}^{-}$strain and the mutant bacterium does not bind to collagen. The appendages contain a globular domain of $3.5 \mathrm{~nm}$ in diameter and a stalk of at least $150 \mathrm{~nm}$ in length and $3.2 \mathrm{~nm}$ in diameter which often show a bend and can be divided into two regions. The length of the stalk domain close the globular domain is very constant $(24 \mathrm{~nm})$. The analysis of the mutant strain complemented with emaA shows the antenna-like appendages on the bacterial surface; some times with a higher coverage than the wild type bacterium. An increase in the number of appendages of the complemented strain correlates with an increase in collagen binding. Inframe deletion analysis indicates that the collagen binding activity is associated with the head domain. A deletion of the whole collagen binding domain reduces the collagen binding activity to null mutant levels. This deletion mutant still expresses surface appendages; however, the globular domain at the distal end of the appendages is missing. The deletion of the third collagen binding motif or an amino acid substitution within this motif (GIAIG) greatly affected the collagen binding activity of EmaA.

Electron tomography studies of whole mount preparations of A. actinomycetemcomitans stained with phosphotungstic acid ( $\mathrm{pH}$ 7.0) are being pursued to obtain more accurate dimensions and higher resolution information on these surface structures. Wild type strains transformed with a plasmid containing emaA are being employed to increase the number of appendages on the cell surface to facilitate the tomography studies. We are collecting double-axis tilt series. All the images in each series are aligned to a common origin and volumes are reconstructed for each series using either a correlation based alignment procedure (script) that we have developed in the SPIDER image processing system [5] or IMOD [6]. Volume merging techniques can be used to reduce the influence of noise [7].

\section{References}

[1] A.H. Kaplan et al. (1989). Rev Infect Dis 11, 46-63.

[2] J.J. Zambon (1985). J Clin Periodontol 12, 1-20 
[3] K.P. Mintz (2004). Microbiology 150, 2677-88.

[4] A. Roggenkamp et al. (2003). J Bacteriol 185, 3735-44.

[5] J Frank et al. (1996) Journal of Structural Biology 116,190-199

[6] J.R. Kremeret al. (1996). Journal of Structural Biology 116, 71-76

[7] T. Ruiz et al. (2001). Journal of Structural Biology. 136, 167-180.
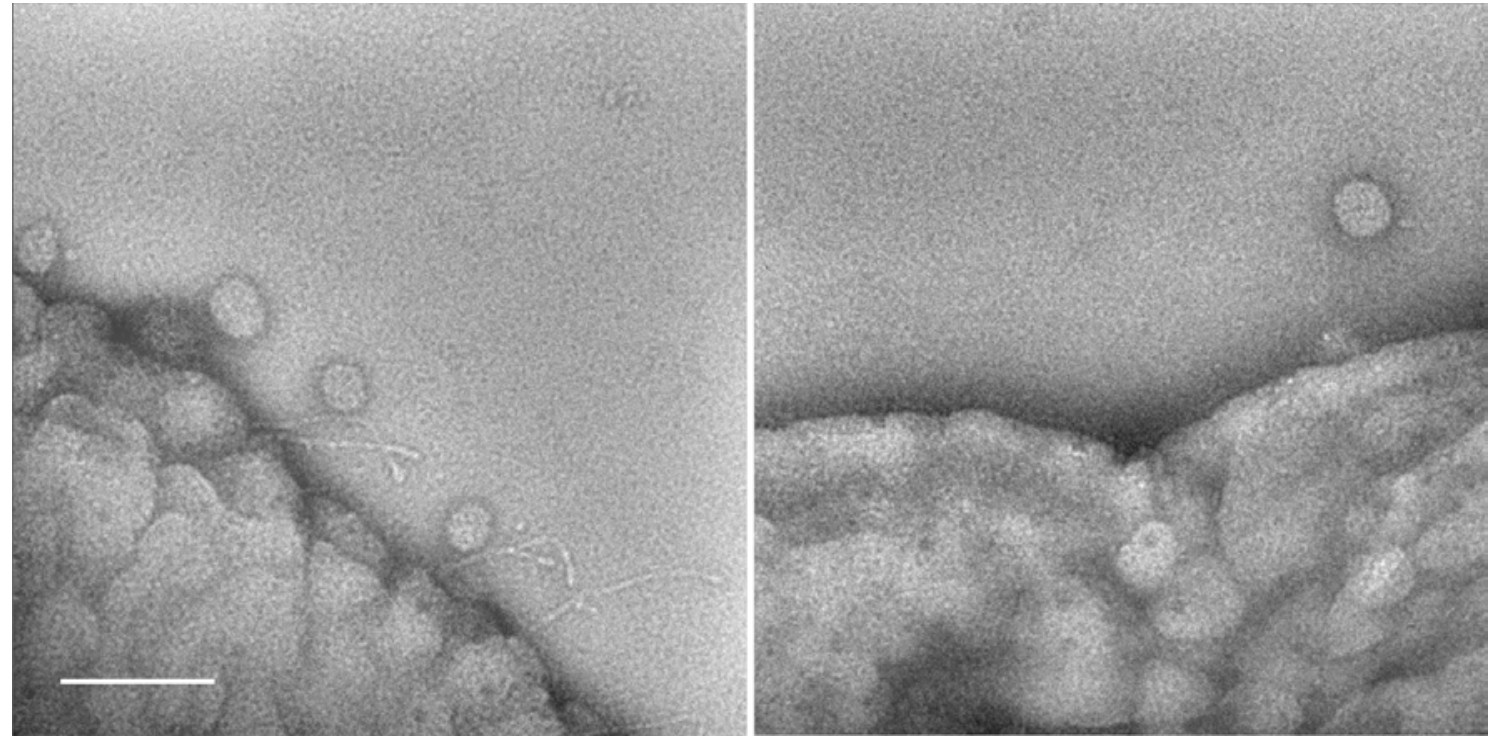

Figure 1. Electron micrographs of $A$. actinomycetemcomitans stained with phosphotugstic acid (PTA) Left: Wild type and Right: $e m a A^{-}$strain. Scale=100nm.
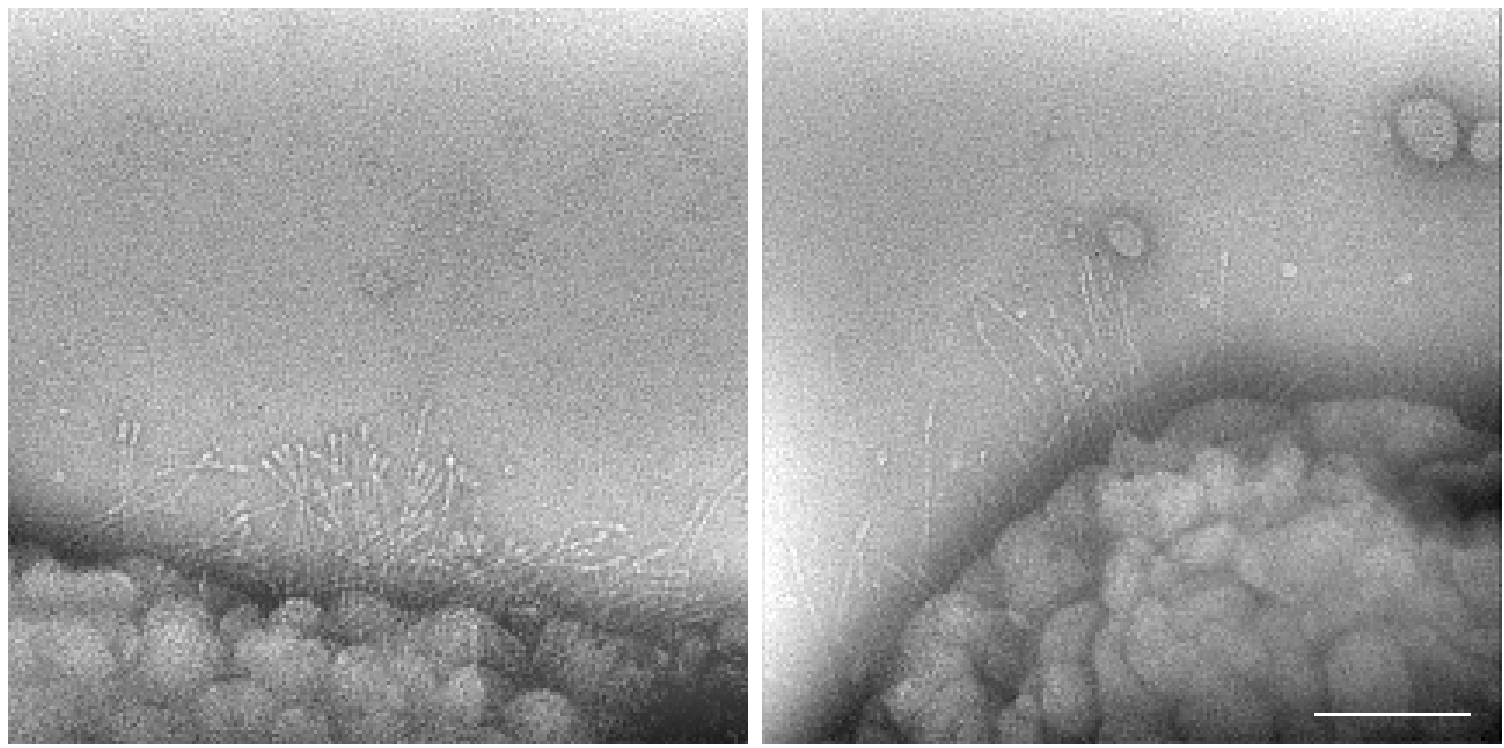

Figure 2. Electron micrographs of $A$. actinomycetemcomitans stained with PTA. Left: emaA ${ }^{-}$strain complemented with the $e m a A$ gene. Right: $e m a A^{-}$strain complemented with the $e m a A$ gene lacking the collagen binding region. Scale $=100 \mathrm{~nm}$. 